\title{
Geometry-aware Partitioning of Complex Domains for Parallel Quad Meshing
}

\author{
Xin Li Wuyi Yu Celong Liu \\ School of Electrical Engineering and Computer Science, Louisiana State University
}

\begin{abstract}
We develop a partitioning algorithm to decompose complex 2D data into small and simple subregions for effective parallel quad meshing. To support effective quad mesh generation, the partitioning reduces to solving an integer quadratic optimization problem with linear constraints. Directly solving this problem is expensive for large-scale data. Hence, we also suggest a more efficient two-step algorithm to obtain an approximate solution. First, we partition the region into a set of cells using $L_{\infty}$ Centroidal Voronoi Tessellation (CVT), then we solve a graph partitioning on the dual graph of this CVT to minimize the total partitioning boundary length, while enforcing the load balancing and each subregion's connectivity. With this decomposition, subregions arcarslaw1940somee distributed to multiple processors for parallel quadrilateral mesh generation. Through thorough comparisons on the quality of the final meshes and the performance of simulations run on these meshes, we show that our decomposition algorithm outperforms existing partitioning approaches by offering better partitioning, and therefore, more simulation-friendly regular meshes.
\end{abstract}

Keywords: Geometry-aware Data Partitioning; Parallel Mesh Generation; Large-scale Regular Meshing.

\section{Introduction}

Mesh generation is a fundamental and critical problem in geometric data modeling and processing. In most scientific and engineering tasks that involve numerical computations or simulations (e.g. solving partial differential equations (PDE) using finite difference methods (FDM), finite element methods $(\mathrm{FEM})$, or finite volume methods $(\mathrm{FVM}))$ on geometric regions or objects, 
the first step of the procedure is always to discretize the geometric data using polygonal or polyhedral meshes. The quality of this tessellation often significantly affect the subsequent computation accuracy, efficiency, and numerical stability. Compared with commonly used irregular tessellations (such as triangular meshes in 2D and tetrahedral meshes in 3D), the regular tessellations (such as quadrilateral meshes in 2D and hexahedral meshes in 3D) are sometimes preferred in finite element analysis or isogeometric analysis in structural mechanics, fluid dynamics simulations, and several other fields, because they demonstrate more efficient and accurate computing [1] and more economic storage.

In recent years, the acquisition or generation of large high-resolution geometric datasets pose new challenges to the design of effective processing algorithms. These big and complex data are expensive to model and analyze using existing sequential algorithms, as the limited CPU and memory are often insufficient to handle billions of elements efficiently. Parallel algorithms utilizing high-performance computers make it possible to solve large and complex problems efficiently on hundreds or thousands of computing clusters and is therefore desirable.

Divide-and-conquer is a natural and effective strategy in parallel mesh generation for large geometric data. The given region or object is first decomposed into a set of solvable and simplified subparts; then each subpart is distributed to a working processor for mesh construction; finally, individually generated meshes are merged to get the final result. Parallel mesh generation strategies such as Delaunay-based methods and advancing front techniques have been used for both triangulation $[2,3,4]$ or tetrahedralization $[5,6]$. Following this general paradigm, in this work we aim to develop a partitioning algorithm on comlex and large-scale 2D regions for parallel quadrilateral mesh generation.

In geometric processing through divide-and-conquer, the partitioning of data often directly dictates the algorithm's efficiency and quality. We formulate three main criteria as follows: (1) The areas of the subregions should be similar; (2) The boundary length of each subregion should be small compared with it's area; and (3) Each subregion should have desirable geometric property, and more specifically for quad meshing, it should have corner angles close to $k \pi / 2, k \in\{0,1,2,3\}$. The first two criteria determine the parallel performance: the balance of work load on different processors, and the communication cost among processors. Efficient parallelization should balance the workload distributed to different processors and should minimize inter- 
processor communication to reduce synchronization and waiting. The third criterion on the subregion's geometry affects the quality of the final quad tessellation. For example, on a square subregion one can generate a quad mesh where every element is uniform and not sheared; but on a triangle subregion, the smallest angle of the resultant quad mesh will inevitably be smaller than the smallest boundary angle of this triangle patch. Hence, for effective quad mesh generation, it is desirable to partition the geometry into subregions whose boundary angles are near $k \pi / 2$ to construct less-sheared quad elements. To incorporate the geometric constraint in data partitioning, however, is sometimes prohibitively expensive. We will discuss this issue in Section 3 and propose a more efficient strategy to tackle it. The three criteria are shown in Figure 1

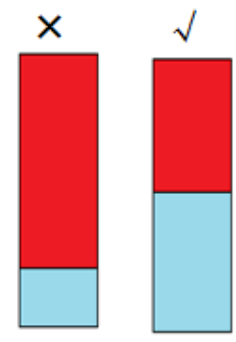

(a)

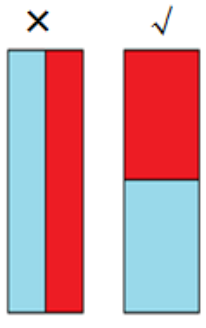

(b)

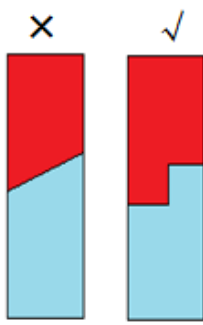

(c)

Figure 1: Three criteria for a good partitioning for (a) balanced workload (b) small communication and (c) small angle deviation to $k \pi / 2$.

After data partitioning, we distribute subregions to different working processors for mesh generation. In our implementation, we use consistent boundary sampling and advancing front for parallel meshing on each subregion. The sub-meshes are finally merged together then a relaxation is performed to get the final result. The main contributions of this paper include:

- We study geometry-aware data partitioning for effective parallel mesh generation, and suggest new models to partition large and complex 2D regions into subparts with desirable geometric shapes for quad meshing. Compared with existing partitioning algorithms, our approach could lead to more efficient parallel processing and higher-quality meshing results.

- We develop a parallel computing framework for quad mesh generation of large-scale 2D regions. It can effectively utilize parallel computational resources to handle big geometric data. We demonstrate that in coastal 
modeling the massive-size coastal terrains and oceans can be discretized effectively.

- We demonstrate that our framework is simulation-friendly. We perform two simulations to show that the meshes generated by our framework can reach higher accuracy than others.

\section{Related Work}

\subsection{Geometric Region Partitioning}

Given a geometric region $M$, a set of components $\left\{M_{i}\right\}$ is a partition of $M$ if (1) their union is $M$, i.e., $\bigcup_{i} M_{i}=M$, and (2) all $M_{i}$ are interior disjoint, namely, $\forall_{i \neq j} M_{i}^{\circ} \cap M_{j}^{\circ}=\emptyset$, where $M_{i}^{\circ}=M_{i} \backslash \partial M_{i}$ is the open set of $M_{i}$. Depending on the geometric processing applications, partitioning techniques consider different criteria accordingly. Thorough surveys on geometric partitioning algorithms have been given $[7,8]$ for computer graphics and geometric modeling applications; some data benchmarks [9] have been built for evaluating these methods in these graphics applications.

Slightly different from the partitioning criteria considered in graphics applications, in order to obtain effective data partitioning for parallel computing, partition strategies can be classified into two categories: extrinsic space partitioning and intrinsic manifold partitioning methods.

We call the first strategy the extrinsic space partitioning method, because it partitions data by partitioning the data's embedding space. For example, data can be decomposed by spatial subdivision or partitioning structures such as quad-tree or octree [10], axis/planes [3], or blocks [11]. In parallel data processing literature, a very popular extrinsic space partitioning strategy is the space filling curve $[12,13]$. The idea is to first fill the $N$-dimensional space with a 1-dimensional curve and establish a bijective map between cells in the space and curve segments passing them. Different regions (cells) in the space are therefore indexed by this curve, and partitioning of the space (therefore, partitioning of data) is obtained by partitioning the curve accordingly. In general, data (space) partitioning using space-filling curves or other extrinsic space partitioning methods is very efficient, as demonstrated in several successful applications, such as computational physics, algebraic multigrid, PDE solving, adaptive mesh refinement [14, 15]. However, algorithms based on spatial partitioning are not suitable to handle data that have complex geometry or nonuniform properties. 
We call the second category the intrinsic manifold partitioning method, and it partitions the data model on its intrinsic tessellation. The data are discretized into a mesh or a graph, where elements or nodes are clustered into subparts directly [16] or recursively [17]. Among this category, an effective and widely used strategy is called graph partitioning [18, 19, 20, 21, 22], which usually produce good-quality partitions with balanced load and reduced communication. Solving the graph partitioning is NP-hard, and several effective strategies include spectral bisection [19], Kerninghan-Lin algorithm [18] and the multi-level scheme [20]. The spectral bisection algorithm [19] uses the spectral information to partition the graph. The eigenvector of the Laplacian matrix is computed to bisect the graph. Spectral Bisection usually produces a good partitioning, however it is very expensive to compute, especially for large matrix. On the other hand, the Kerninghan-Lin algorithm [18] is a fast heuristic scheme. It starts with an initial bipartition of the graph, then iteratively swaps a subset of vertices from each part to reduce the energy. This algorithm is fast, however, the initial partition is critical but often not easy to obtain. The multi-level method, including the widely adopted algorithm/software METIS [20], uses a three-phase scheme: first, the graph is simplified to a coarse graph; then a partitioning is performed on the coarsened graph; finally, the partitioning is modified during the progressive graph refinement. These existing graph partitioning algorithms focus on only tackling the load balancing and communication reduction issues. However, only considering these two criteria is insufficient. Incorporating extra geometric constraints is often critical in geometric modeling applications.

The Medial Axis Domain Decomposition (MADD) algorithm [23] merges triangles to eliminate small angles then solves a graph partitioning on dual graph of the merged mesh. Subregions constructed using MADD partitioning usually possess larger corner angles than METIS. For our problem, it is desirable to have perpendicular corner angles. Therefore, a geometric term to incorporate this angle constraint can be formulated and included into the graph partitioning model. Furthermore, an additional connectivity constraint is needed to ensure each subregion form only one connected component. Exactly solving this graph partitioning problem with these extra geometric constraints is NP-hard; hence, a computationally efficient approximation algorithm will be desirable. 


\subsection{Quadrilateral Mesh Generation}

Quadrilateral mesh generation has been studied in computer graphics and geometric modeling fields. Quad meshes can be constructed through either the indirect or direct approaches. The indirect approaches first generate an intermediate structure/tessellation that can be easily constructed, e.g., a triangle mesh, then convert it into a quadrilateral mesh [24, 25]. One major limitation of this method is that the layout of the unstructured elements in the intermediate tessellation determines the layout of final quad mesh, and there are usually a large number of singularities (i.e., non-valance- 4 vertices) in the resultant quad meshes, which are usually undesirable for efficient simulation. The direct approaches construct quads on the model directly. Related techniques include quad-tree ([26]), template-guided decomposition ([27]) and advancing front $([28])$. A related problem is the quadrilateral mesh generation on curved surfaces. [29] gave a good survey on this topic. However, the curved geometry pose extra challenges in quad mesh generation and many recent surface quad meshing algorithms $[30,31]$ use a cross frame field to guide the construction of the quad meshes. Finding an optimal cross frame field reduces to nonlinear integer programming, which is computationally expensive for large-scale geometric regions. Another related problem is the quad layout patch construction [32]. Its goal is to partition a surface into topologically rectangle patches, upon which quad meshes can be constructed. In this paper, we didn't adopt this strategy, because the topological constraint on restricting subregions to be "4-sided polygons" is very expensive to enforce.

\section{Region Partitioning}

Data partitioning is the first step in a divide-and-conquer framework for parallel computational models. A good partitioning with balanced workload and small interprocess communication helps improve computational efficiency. Furthermore, in our parallel meshing problem, a good data partitioning is also critical in generating high-quality quad elements. We use three criteria to quantitatively evaluate a partitioning: workload balance, total separator length, and separator angle deviation. Graph Partitioning is a suitable strategy to solve our partitioning, because it can systematically model and optimize these criteria. In this section, we will first introduce the related notation (Sec. 3.1) and formulation of the three criteria (Sec. 3.2, 3.3 and 3.4) and the Connectivity Constraints (Sec. 3.5), then propose our algorithm in Sec. 3.6. 


\subsection{Notation}

Given a tessellation $M=\left(V^{M}, E^{M}, F^{M}\right)$ of a 2 D region, where $V^{M}, E^{M}, F^{M}$ are the sets of vertices, edges, and faces (cells) respectively, let $G=\left(V^{G}, E^{G}\right)$ denotes its dual graph, where $V^{G}, E^{G}$ are the node and arc sets respectively. A node $v \in V^{G}$ corresponds to a cell of $M$. Two nodes $v_{1}, v_{2} \in V^{G}$ are connected by an arc if their corresponding cells are adjacent, namely, share an edge. Therefore, each arc of $E^{G}$ also corresponds to an edge in $E^{M}$. The weight of a node $v \in V^{G}$ is defined to be the area of its associated cell $f \in F^{M}$, and an arc's weight is defined as the length of its associated edge. The partitioning on $G$ can be computed on the dual graph $G$. A $k$-way $G r a p h$ Partitioning divides $V^{G}$ into $k$ connected components, each of which is a subregion that will be processed individually. In practice, $k$-way partitioning is usually solved through recursive bisection [20]. Hence, we recursively partition $G$ into two sub-graphs $G_{0}=\left(V^{G_{0}}, E^{G_{0}}\right)$ and $G_{1}=\left(V^{G_{1}}, E^{G_{1}}\right)$, where $V^{G_{1}}=V^{G} \backslash V^{G_{0}}$. This also partitions cells in the original tessellation $M$ into two sets $M_{0}$ and $M_{1}$, if an edge $e \in M$ is shared by two cells $f_{i}, f_{j}$ from distinct subsets, $f_{i} \in M_{0}, f_{j} \in M_{1}$, then edge $e$ is called a separator.

For each node $v_{i}^{G} \in V^{G}$, we assign a variable $x_{i}$,

$$
x_{i}=\left\{\begin{array}{ll}
0, & \text { if } v_{i}^{G} \in V^{G_{0}} \\
1, & \text { if } v_{i}^{G} \in V^{G_{1}}
\end{array} .\right.
$$

Then for each $\operatorname{arc} e_{i j}^{G}=\left[v_{i}^{G}, v_{j}^{G}\right]$, we assign a variable $y_{i j}=x_{i}-x_{j}$ :

$$
y_{i j}=\left\{\begin{array}{ll}
1 \text { or }-1, & \text { if } v_{i}^{G} \text { and } v_{j}^{G} \text { are in two sub-graphs } \\
0, & \text { otherwise }
\end{array} .\right.
$$

We have $\mathbf{y}=\mathbf{U} \mathbf{x}$, where $\mathbf{x}$ and $\mathbf{y}$ are node and arc variable vector respectively, and $\mathbf{U}$ is a $\left|E^{G}\right| \times\left|V^{G}\right|$ matrix. With these variables, we formulate the three criteria as follows.

\subsection{Workload Balance}

Load balancing refers to the practice of distributing approximately equal amounts of work among tasks, so that all tasks are kept busy all of the time. Unbalanced workload between working processors leads to inefficiency in parallel computing, as the slowest task often determines the overall running time. In our problem, the workload on each working processor can be estimated by the area of each subregion to mesh. On the dual graph, the 
subregion area can be calculated using the sum of weights of nodes in the corresponding subgraph.

A balanced partitioning should avoid big area difference between subregions. Hence, it is formulated as the following constraint:

$$
c_{1} \leq \mathbf{x}^{T} \mathbf{w}_{v}-c \leq c_{2},
$$

where $\mathbf{x}=\left(x_{1}, x_{2}, \ldots, x_{n}\right)^{T}$ is the variable vector, $\mathbf{w}_{v}=\left(w_{v_{1}}, w_{v_{2}}, \ldots, w_{v_{n}}\right)^{T}$ is the node weight vector, $c=\frac{1}{2} \sum_{i} w_{v_{i}}$, and $c_{1}, c_{2}$ are the constant thresholds (in our experiments, $c_{1}=c_{2}=0.1 c$ ).

After a $k$-way partitioning is obtained, its workload balance can be evaluate by the ratio between the areas of the largest and smallest subregions:

$$
R_{W}=A_{\max } / A_{\min }
$$

where $A_{\max }$ and $A_{\min }$ are the areas of the largest and smallest subregions respectively. $R_{W}$ close to 1 means better workload balance.

\subsection{Total Separator Length}

In parallel computing, inter-process communication means overhead. A smaller total separator length usually indicates less communication cost. In our geometric data partitioning, a smaller separator length also indicates (1) smoother subregion boarder lines, (2) better geometric compactness of subregions, and (3) more efficient post-processing refinement (see Section 4). Therefore, it is desirable to minimize the total separator length

$$
L_{S}=\mathbf{y}^{T} \mathbf{W}_{e} \mathbf{y}=\mathbf{x}^{T} \mathbf{U}^{T} \mathbf{W}_{e} \mathbf{U} \mathbf{x}
$$

where $\mathbf{y}=\left(y_{e_{1}}, y_{e_{2}}, \ldots, y_{e_{n}}\right)^{T}$ is the edge variable vector, $\mathbf{W}_{e}=\operatorname{diag}\left(w_{e_{1}}, w_{e_{n}}, \ldots, w_{e_{n}}\right)$ is a diagonal matrix composed of arc weights.

With the above two criteria, solving a graph partitioning can be formulated as:

$$
\begin{array}{ll}
\min & \mathbf{x}^{T} \mathbf{U}^{T} \mathbf{W}_{e} \mathbf{U} \mathbf{x}, \\
\text { subject to } & c_{1} \leq \mathbf{x}^{T} \mathbf{w}_{v}-c \leq c_{2}, \\
& x_{i} \in\{0,1\} .
\end{array}
$$

Optimizing problem (4) is NP-hard. For large data, multilevel schemes such as METIS [20] have been widely adopted to obtain good approximate solutions. Figure 2 illustrates a simple example. The input tessellation $M$ 
is shown in (a) and the partitioning result by METIS [20] is shown in (b). The two partitioned subregions are colored in green and orange, respectively. We can see that the two subregions have the same area with total separator length minimized.

Minimizing the total separator length will makes the boundary of subregions straight, and it can increase the compactness of each subregion. On each subregion $M_{i}$, the compactness can be measured by the ratio between the boundary separator's length of $M_{i}$ and the area of $M_{i}$. Globally, we can compute an average compactness ratio,

$$
\hat{R}_{C}=\frac{L_{S}}{k A_{M}}
$$

where $k$ is number of subregions, and $A_{M}$ is the total area of the region. Smaller $\hat{R}_{C}$ comes from a smaller $L_{S}$ and indicates better compactness.

\subsection{Separator Angle Deviation}

Solving the graph partitioning formulated in Eq. (4) will result in balanced area and minimized total separator length. However, in many geometric processing tasks, constraints on the geometry of subregions are important. In our quad meshing problem, ideally, each subregion should look like a square. Less strictly, since we use the advancing front technique to generate quad meshes (Section 4), it is desirable to have angles between separators close to $\frac{k \pi}{2}$. Therefore, we include a new separator angle term into graph partitioning to penalize each such angle's deviation from $\frac{k \pi}{2}$.

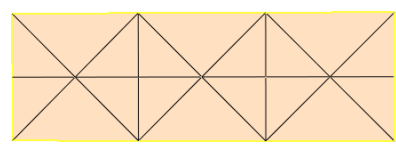

(a)

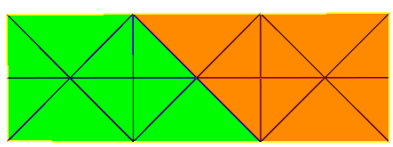

(b)

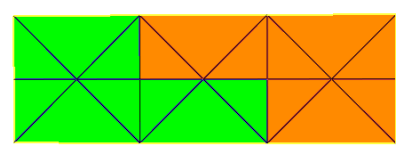

(c)

Figure 2: Graph Partitioning on a simple example. (a) The original mesh; (b) the two (green and orange) subregions obtained by METIS [20]; (c) the partitioning result with separator angle deviation considered.

Angle Deviation Function. Consider the original tessellation $M$ of the given $2 \mathrm{D}$ region, suppose two edges $e_{i}, e_{j} \in E^{M}$ share a vertex $v$ and form an angle $\theta_{i, j}$. For concise representation, in the following, we use $\operatorname{Inc}(i, j)=1$ to 
denote $e_{i}$ and $e_{j}$ are incident, and $\operatorname{Inc}(i, j)=0$ means they are not incident. We define an angle deviation function

$$
\delta_{\theta_{i, j}}=\left\{\begin{array}{cl}
\min _{k}\left\{\left|\theta_{i, j}-\frac{k \pi}{2}\right|, k \in\{0,1,2,3\}\right\}, & \text { if } \operatorname{Inc}(i, j)=1 \\
0, & \text { if } \operatorname{Inc}(i, j)=0
\end{array}\right.
$$

to describe the deviation from angle $\theta_{i, j}$ to the nearest $\frac{k \pi}{2}$ angle.

Accumulated separator angle deviation can then be formulated as

$$
D_{\theta}=\mathbf{y}^{T} \mathbf{W}_{\theta} \mathbf{y}=\mathbf{x}^{T} \mathbf{U}^{T} \mathbf{W}_{\theta} \mathbf{U} \mathbf{x},
$$

where $\mathbf{y}=\left(y_{e_{1}}, y_{e_{2}}, \ldots, y_{e_{n}}\right)^{T}$ is the edge variable vector,

$$
\mathbf{W}_{\theta}=\left(\begin{array}{ccccc}
0 & \delta_{\theta_{1,2}} & \delta_{\theta_{1,3}} & \ldots & \delta_{\theta_{1, n}} \\
\delta_{\theta_{2,1}} & 0 & \ldots & \ldots & \delta_{\theta_{2, n}} \\
\ldots & \ldots & \ldots & \ldots & \ldots \\
\delta_{\theta_{n, 1}} & \ldots & \ldots & \ldots & 0
\end{array}\right)
$$

is an $\left|E^{G}\right| \times\left|E^{G}\right|$ matrix storing deviation angles $\delta_{\theta_{i, j}}$. This angle deviation matrix $\mathbf{W}_{\theta}$ can be precomputed by traversing all the edge pairs of the tessellation $M$ once.

Furthermore, we can show that Eq. (7) will evaluate and only evaluate the angle deviation between adjacent separators. Suppose two edges $e_{i}=$ $[u, v], e_{j}=[u, w]$ are incident separators, sharing vertex $u$. Then, (1) $y_{e_{i}} \neq 0$ and $y_{e_{j}} \neq 0$, and (2) $v$ and $w$ belong to the same subregion and have a same indicator value, $x_{v}=x_{w}$. From (1) and (2), it is not difficult to see that $y_{e_{i}}$ and $y_{e_{j}}$ are both -1 or both +1 . Therefore, a non-negative contribution $\delta_{\theta_{i, j}}$ will be added to the accumulated separator angle deviation $D_{\theta}$. To obtain a geometrically desirable partitioning, we can minimize an objective function of the separator angle deviation term (7) plus the total separator length term (3), subject to the workload balance constraint (1).

Finally, after the partitioning, we can numerically evaluate the average separator angle deviation:

$$
\hat{\delta}_{\theta}=\frac{1}{N_{C}}\left(\sum_{y_{i j} \neq 0, \operatorname{Inc}(i, j)=1} \delta_{\theta_{i, j}}\right),
$$

where $N_{C}$ is the total number of corners formed by incident separators. The smaller $\hat{\delta}_{\theta}$ is, the better. 


\subsection{Connectivity Constraints}

Currently we have formulated the three criteria. To guarantee the result is a bipartitioning, we need to impose the connectivity constraint: nodes in each subregion should form one connected component. This connectivity constraint is often not explicitly considered in existing graph partitioning literature, sometimes due to its complexity and sometimes due to unnecessity of the target application. In our problem discussed here, however, without explicitly enforcing this, although minimizing total separator length tends to penalize the partition that produces multiple disjoint subsets, a locally optimal solution that has multiple connected components in one subregion could be obtained. An example is illustrated in Fig. 7 (a).

To enforce connectivity of each subregion, we can formulate the following explicit constraint. Given a (dual) graph $G=\left(V^{G}, E^{G}\right)$, for any pair of non-adjacent nodes $u, v$, we define a node set $S \subset V \backslash\{u, v\}$ to be a node-cut set that separates $u$ and $v$, if there is no path between $u$ and $v$ after $S$ is removed from $G$. For example, in the graph shown in Fig 3, for node pair $\{1,4\},\{2,5,7\}$ is a node-cut set and $\{3,6,8\}$ is another node-cut set. For $\{u, v\}$ that $[u, v] \notin E^{G}$, we define $\Gamma(u, v)$ to be the set consisting of all the node-cut sets of $\{u, v\}$. In this example, $\Gamma(1,4)=$ $\{\{2,5,7\},\{2,5,8\},\{2,6,7\},\{2,6,8\},\{3,5,7\},\{3,5,8\},\{3,6,7\},\{3,6,8\}\}$.

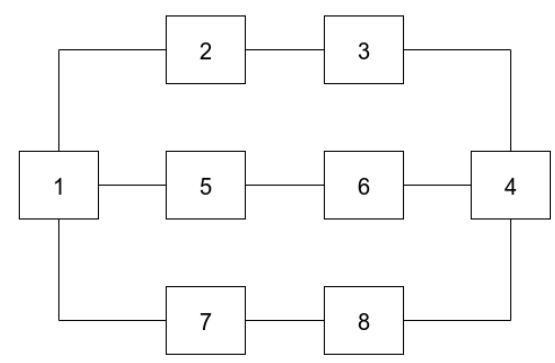

Figure 3: An example graph for connectivity constraint.

The connectivity constraint can be described as: between each pair of nodes $u, v$ that are in the same subregion, any node-cut set in $\Gamma(u, v)$ must have at least one node being assigned to this subregion. Using the binary variable $x_{i}$ defined previously, the connectivity constraints can be formulated as a set of linear constraints. For any two non-adjacent nodes in subgraph $G_{1}$ :

$$
\sum_{w \in S} x_{w} \geq x_{u}+x_{v}-1, \forall S \in \Gamma(u, v), \text { for } \forall x_{u}=x_{v}=1,[u, v] \notin E^{G},
$$


meaning that every node-cut set must have at least one node being assigned as 1 . Similarly, for any two non-adjacent nodes in subgraph $G_{0}$ :

$$
\sum_{w \in S} x_{w} \leq x_{u}+x_{v}+|S|-1, \forall S \in \Gamma(u, v), \text { for } \forall x_{u}=x_{v}=0,[u, v] \notin E^{G} .
$$

These constraints ensure that there is at least one path connecting nonadjacent nodes $u$ and $v$ if they are grouped to a same subgraph.

By incorporating both the separator angle deviation (Eq. 6) and connectivity constraints (Eqs. 9,10) into graph partitioning, the quadratic integer programming problem with linear constraints is finally formulated as:

$$
\begin{array}{cc}
\lambda_{1} L_{S}+\lambda_{2} D_{\theta} \\
\text { minimize } \\
\text { subject to } & \mathbf{0} \leq \mathbf{x} \leq \mathbf{1}, \text { and Constraints (1), (9), (10) },
\end{array}
$$

where $\lambda_{1}$ and $\lambda_{2}$ are weighting factors (in our experiments, we simply set $\left.\lambda_{1}=\lambda_{2}=1\right), L_{S}$ and $D_{\theta}$ are separator length term (3) and separator angle deviation term (7).

We call the partition obtained by optimizing this problem the Exact Optimization Geometric-aware Partitioning (EOGP). This problem (11) can be solved through a branch-and-bound algorithm. In our implementation, we use the open-source Basic OpeN-source Mixed INteger (BONMIN) solver from [33] to obtain a solution. To solve mixed integer non-linear programming problems, BONMIN allows one to choose optimization strategies including branch-bound, outer approximation (OA), Quesada Grossman branchcut, and Hybrid OA based branch-cut.

Figure 2 (c) shows the solution of this new graph partitioning. With the minimization of separator angle deviation and the enforcement of connectivity constraints, a partitioning more suitable for quad mesh generation is obtained. However, because (1) incorporating separator angle deviation significantly increase the numbers of non-zero cross multiplication of indicator variables, and (2) the enforcement of connectivity introduces an exponential number of linear constraints. Solving such a new problem becomes prohibitively expensive for even moderately large problem.

\subsection{Our Two-Step Partitioning Algorithm}

We proposed a two-stage partitioning scheme to incorporate the two new constraints discussed in the last section. A key observation this idea based upon is that: if the cells of the initial tessellation has near-square geometry, 
then the partition performed on the dual graph of this tessellation tends to have smaller separator angle deviation. Hence, first, we use $L_{\infty}$-CVT to generate a tessellation with cells similar to squares (Sec. 3.6.1); second, we solve our graph partitioning on this tessellation to get a set of subregions with balanced workload and small total separator length, with connectivity constraints enforced on the subgraphs during their refinement.(Sec. 3.6.2) We call this algorithm a $L_{\infty}-C V T-G P$ algorithm for short, and the idea is illustrated in Fig. 4.

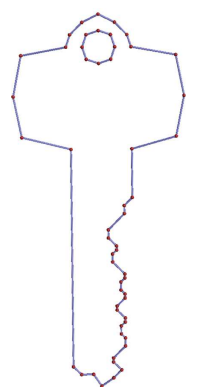

(a)

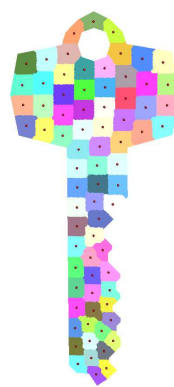

(b)

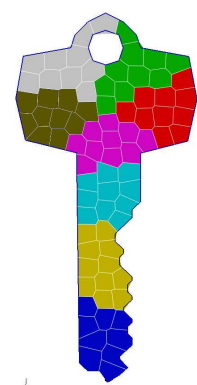

(c)

Figure 4: Partitioning a 2D "Key" Region for Parallel Quad Mesh Generation. (a) The input 2D boundary; (b) $L_{\infty}$-norm CVT on the input boundary; (c) our partitioning result, with different subregions indicated using different colors.

\subsection{1. $L_{\infty}$ Centroidal Voronoi Tessellation}

The Voronoi diagram is a fundamental geometric structure widely used in various fields, especially in geometric modeling and computer graphics. A

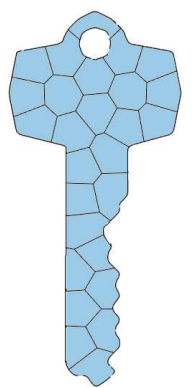

(a) $p=4$

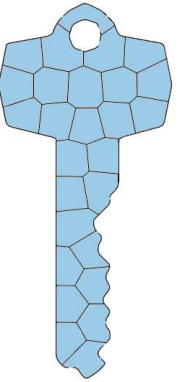

(b) $p=6$

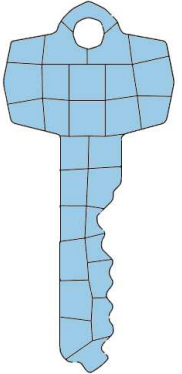

(c) $p=8$

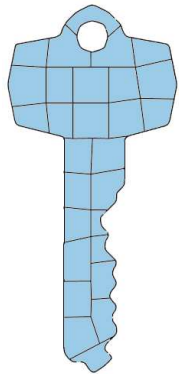

(d) $p=10$

Figure 5: $L_{p}$-CVT using different $p$ values. The results are similar when $p>=8$. In our experiments, we use $L_{8}$-CVT to approximate $L_{\infty}$-CVT. 
2D Voronoi Diagram of a given set of distinct points $\mathbf{X}=\left\{\mathbf{x}_{i}\right\}_{i=1}^{n}$ in $\mathbf{R}^{2}$ is defined by a set of Voronoi cells $\left\{\Omega_{i}\right\}_{i=1}^{n}$ where

$$
\Omega_{i}=\left\{\mathbf{x} \in \mathbf{R}^{2} \mid\left\|\mathbf{x}-\mathbf{x}_{i}\right\| \leq\left\|\mathbf{x}-\mathbf{x}_{j}\right\|, \forall j \neq i\right\} .
$$

These points $\mathbf{X}$ are called sites. Each Voronoi cell $\Omega_{i}$ is the intersection of a set of half-planes. A Clipped Voronoi Diagram for the sites $\mathbf{X}$ within a given $2 \mathrm{D}$ domain $\Omega$ is the intersection of the Voronoi Tessellation and the domain $\Omega$, denoted by $\left\{\left.\Omega_{i}\right|_{\Omega}, i=1, \ldots, n\right\}$, where

$$
\left.\Omega_{i}\right|_{\Omega}=\left\{\mathbf{x} \in \Omega \mid\left\|\mathbf{x}-\mathbf{x}_{i}\right\| \leq\left\|\mathbf{x}-\mathbf{x}_{j}\right\|, \forall j \neq i\right\} .
$$

In other words, $\left.\Omega_{i}\right|_{\Omega}=\Omega_{i} \cap \Omega$. The Centroidal Voronoi Tessellation (CVT) is that each site of the Voronoi cell is coincident to this cell's centroid.

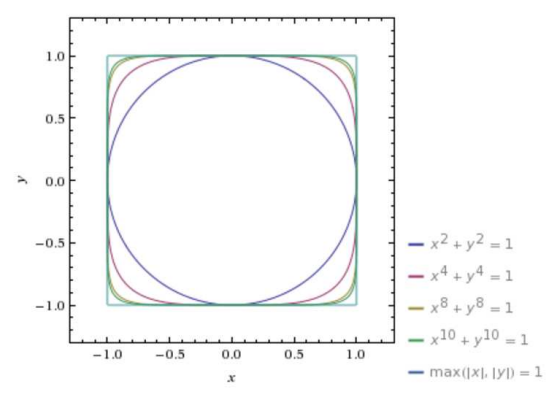

Figure 6: The trajectory of $L_{p}(x, y)=1$ with different $p$ values. With the increase of $p$ the trajectory gradually approximates the unit square. $L_{\infty}(x, y)=1$ gives a square.

The $L_{p}$ Centroidal Voronoi Tessellation Energy [34] can be defined as:

$$
F(\mathbf{X})=F\left(\left[\mathbf{x}_{1}, \mathbf{x}_{2}, \ldots, \mathbf{x}_{n}\right]\right)=\sum_{i} \int_{\Omega_{i} \cap \Omega}\left\|\mathbf{y}-\mathbf{x}_{i}\right\|_{p}^{p} d \mathbf{y}
$$

where $\|\mathbf{z}\|_{p}=\left(\sum_{j}\left|z_{j}\right|^{p}\right)^{\frac{1}{p}}, z_{j}$ is the $j$-th coordinate of a $2 \mathrm{D}$ point $\mathbf{z}$. Figure 6 shows the trajectory with different $p$. With the increase of $p$, the trajectory approximates to the square. The trajectory of $L_{\infty}$ is a perfect square, where the $L_{\infty}$ norm of a $d$-dimensional vector $\mathbf{z}$ is the maximal component in $\mathbf{z}$, $\|\mathbf{z}\|_{\infty}=\max _{j}\left|z_{j}\right|$. For efficient CVT computation, we choose a sufficiently large $p$ to approximate the $L_{\infty}$ norm. This also allows us to efficiently compute the gradient of $F(\mathbf{X})$ of Eq. (13). We test $L_{p}$-CVT on the key model using different $p$ values. And as the results illustrated in Figure 5, when 
$p>8$, the difference of $L_{p}$-CVT energy becomes very small, so we use $L_{8^{-}}$ CVT to approximate $L_{\infty}$-CVT in all our experiments. Since we use $L_{8}$ which is smooth, the optimization of CVT energy $F$ can be solved efficiently using the quasi-Newton BFGS solver [35].

In summary, the algorithm to compute the $L_{p}$-CVT on the input $2 \mathrm{D}$ region $\Omega$ has four steps.

1. Get a uniformly sampled sites set $\mathbf{X}$. In our implementation, we simply embed $\Omega$ on a spatial grid, the grid points inside $\Omega$ are the initial sites.

2. Use the sweeping line algorithm [36] to construct the initial Voronoi Diagram.

3. Get the $L_{\infty}$-CVT tessellation by optimizing CVT energy.

4. Clip the CVT using $\partial \Omega$ to get the $L_{\infty}$-CVT of $\Omega$.

\subsubsection{Graph Partitioning Based on $L_{\infty}-C V T$}

After solving the $L_{\infty}$-CVT, we get a tessellation $\widetilde{M}$ of the $2 \mathrm{D}$ region. Then on $\widetilde{M}$ 's dual graph $\widetilde{G}$, we solve the graph partitioning formulated in Eq. (4). Following the heuristics used in [20], instead of explicitly enforcing the large number of connectivity constraints in Eqs. $(9,10)$, we can adopt a region growing examination to check the connectivity of each subregion, disconnected elements will be grouped into the other subregion automatically. During our optimization, after every $K$ iterations, we perform such

an examination and update on $\widetilde{G}$. In practice, this strategy is efficient and effective in enforcing the connectivity of subregions.

\subsection{Discussion}

\subsubsection{Comparison between $L_{\infty}-C V T-G P$ and EOGP}

We compare our $L_{\infty}$-CVT-GP algorithm with the Exact Optimization Geometric-aware Partitioning (EOGP) algorithm on the key model. The results are shown in Fig. 7. (a) shows the partitioning result without using connectivity constraint, which is undesirable for mesh generation. (b) is the result from EOGP. (c) is $L_{\infty}$-CVT-GP algorithm result. Table 1 shows the statistics. Compared with EOGP (which yields the best result), the $L_{\infty}$-CVT-GP algorithm generates comparable result to the optimization algorithm in workload balancing ratio, average compactness and average separator angle deviation, and it only uses about 10\% running time of EOGP. Therefore, $L_{\infty}$-CVT-GP is more suitable for large-sized geometric data. 


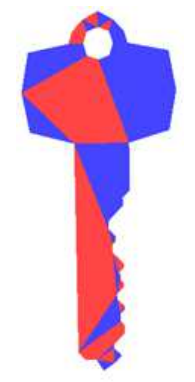

(a)

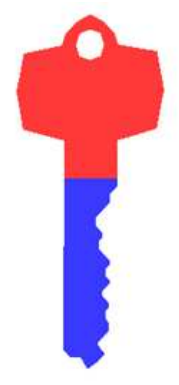

(b)

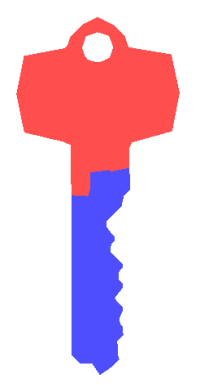

(c)

Figure 7: Partitioning Results on the Key Model using Optimization approach and our $L_{\infty^{-}}$ CVT-GP algorithm. (a), (b) Partitioned by Optimization without and with connectivity constraints respectively; (c) partitioned by our two-step $L_{\infty}$-CVT-GP algorithm.

Table 1: Partitioning statistics for Exact Optimization Geometric-aware Partitioning (EOGP) and our $L_{\infty}$-CVT-GP algorithms on the key model into 2 subregions. $R_{W}, \hat{R}_{C}$, and $\hat{\delta}_{\theta}$ are the workload balance ratio, average compactness, and average separator angle deviation. $t$ is the running time in seconds.

\begin{tabular}{|c|c|c|c|c|}
\hline & $R_{W}$ & $\hat{R}_{C}$ & $\hat{\delta}_{\theta}$ & $t$ \\
\hline EOGP & 1.11 & 0.42 & 0.06 & 385 \\
\hline$L_{\infty}$-CVT-GP & 1.13. & 0.43 & 0.06 & 42 \\
\hline
\end{tabular}

\subsubsection{Comparison among METIS, $L_{\infty}-C V T-G P$ and Direct $C V T$}

We test different partitioning results on the Key model using (a) original graph partitioning by METIS, (b) geometry-integrated graph partitioning solver introduced in Sections 3.4 and 3.5, and (c) this two-step $L_{\infty}$-CVTGP algorithm. We also evaluate the workload balancing ratio $R_{W}$, average compactness $\hat{R}_{C}$, and average separator angle deviation $\hat{\delta}_{\theta}$ on these partition results. Using these three partitioning methods, $R_{W}$ are 1.13, 1.14, and 1.18, respectively; $\hat{R}_{C}$ are $1.11,1.15$, and 1.18 , respectively, and $\hat{\delta}_{\theta}$ are $0.26,0.12$, and 0.15 , respectively.

As expected, the graph partitioning without geometric constraint focuses on workload balance and separator lengths, and gets best $R_{W}$ and $\hat{R}_{C}$, but very bad angle deviation. The expensive geometry-integrated graph partitioning produces smallest angle deviation. This two-step $L_{\infty}$-CVT-GP produces results with slightly worse $R_{W}, \hat{R}_{C}$, and $\hat{\delta}_{\theta}$ than that from the geometry-integrated graph partitioning. But its separator angle deviation is significantly better than the original graph partitioning, and its speed is 
significantly faster than geometry-integrated graph partitioning. More thorough comparisons will be given in Section 5 .

Through the $L_{\infty}$-CVT, we can also directly obtain a region partitioning. Is such a partitioning sufficient, so that we no longer need to further solve a graph partitioning? This section illustrates that the two-step $L_{\infty^{-}}$ CVT-GP algorithm usually leads to a better data partition for the parallel meshing problem. One observation is that: with the increase of the number of Voronoi sites/cells, the decomposition from $L_{\infty}-C V T$ will become more uniform (better workload balancing) and more square-like (smaller separator angle deviation). We have performed extensive experiments on $L_{\infty}$-CVT to verify this. Table 2 is the partitioning statistics on the key and the pipe model. If we directly evaluate the quality of the partitioning suggested by the CVT decomposition, when the number of Voronoi cells increases from 16 to 128 , the workload balancing ratio $R_{W}$ (Eq. (2)) reduces from 1.65 to 1.23, while the average separator angle deviation $\hat{\delta}_{\theta}$ also reduces from 0.26 to 0.18 .

Table 2: Partitioning on the Key and Pipe models using direct CVT versus using our CVT-GP algorithm. $N_{S}$ is the number of subregions, $R_{W}, \hat{R}_{C}$, and $\hat{\delta}_{\theta}$ are the workload balance ratio, average compactness, and average separator angle deviation.

\begin{tabular}{|c|c|c|c|c|c|c|}
\hline & \multicolumn{3}{|c|}{ Key } & \multicolumn{3}{c|}{ Pipe } \\
\hline & \multicolumn{3}{|c|}{ CVT/CVT-GP } & \multicolumn{3}{c|}{ CVT/CVT-GP } \\
\hline$N_{S}$ & 16 & 32 & 128 & 32 & 64 & 256 \\
\hline$R_{W}$ & $1.65 / 1.15$ & $1.45 / 1.24$ & $1.23 / 1.11$ & $1.81 / 1.31$ & $1.65 / 1.31$ & $1.41 / 1.27$ \\
\hline$\hat{R}_{C}$ & $1.21 / 1.11$ & $2.21 / 2.01$ & $9.41 / 9.24$ & $1.82 / 1.77$ & $4.04 / 3.88$ & $18.11 / 17.84$ \\
\hline$\hat{\delta}_{\theta}$ & $0.26 / 0.21$ & $0.21 / 0.16$ & $0.18 / 0.17$ & $0.23 / 0.20$ & $0.18 / 0.15$ & $0.14 / 0.12$ \\
\hline
\end{tabular}

We have the following observations.

- For a $k$-way partition, direct partitioning through a $L_{\infty}$-CVT decomposition with $k$ cells will lead to a worse partitioning result than our two-step algorithm (which first generates $n$ cells $(n>k$ ) then performs a graph partition to get $k$ subregions).

- In order to obtain a partition with similar $R_{W}$ and $\hat{\delta}_{\theta}$, direct $L_{\infty}$-CVT decomposition should use more sites. But this will increase the total separator length, resulting in bigger overhead and more singularities.

And we conclude that the $L_{\infty}$-CVT-GP algorithm offers a better partition than the direct $L_{\infty}$-CVT decomposition. More comparisons will be given in 
Section 5 .

\section{Quad Mesh Generation}

\subsection{Parallel Quad Meshing on Subregions}

After the entire 2D region is partitioned, subregions can be sent to different processors for simultaneous mesh generation. Different quad meshing techniques can be applied on the sub-regions. One requirement is to make sure the neighbouring subregion boundaries should have consistent vertices. We use the advancing front technique [28] to tile the interior regions with quads. To ensure the individually constructed sub-meshes can be composed directly, we need to sample boundary vertices consistently on the shared edge of adjacent subregions. We use a simple sampling scheme: first, we compute an average edge length $\bar{l}$ from input boundary line segments, then on each interior partitioning boundary curve $S$ we evenly sample $\frac{l_{S}}{2 l}$ points, where $l_{S}$ is the length of $S$. To ensure the valid generation of a full quad mesh, the number of the points on the subregion boundary must be even [28], therefore, each interior separator line segments is subdivided into two to ensure the number of sampled boundary vertices is even.

The advancing front algorithm initiates a wave front from the boundary of each subregion, along which quadrangles are constructed inwards until all empty regions are tiled. We implement the advancing front following [28]. The wave front propagates until the front has 6 or fewer vertices, by when a template is used to finish the quad mesh generation. Readers are referred to [28] for details.

\subsection{Post-processing after Composition}

After composing meshes of subregions, we perform a Laplacian relaxation on vertices near separators to improve the smoothness of the orientations of mesh elements on the partitioning boundary. Each vertex moves towards the mass center of its neighboring vertices. Since our partitioning algorithm minimizes the total separator length, this relaxation only applies to a small number of vertices and takes a short time to process. In our experiments, up to 50 iterations are applied to each vertices within a five-ring buffer zone surrounding separators. In our implementation, we didn't parallelize this post-processing. But naturally, this refinement can be easily parallelized if needed. 


\section{Experimental Results}

To evaluate the effectiveness of generated quadrilateral meshes, we perform two types of measurements. One is to directly evaluate the mesh quality, including the element distortion and singularity number (this Section), and the other is to compare the efficiency of scientific simulations running on different meshes (Section 6).

We perform experiments on our high performance computing clusters consisting of 128 nodes. Each node has two 2.6GHz 8-Core Xeon 64-bit Processors and 32GB memory. Five datasets have been tested: a key model with $21.6 \mathrm{M}$ boundary segments and 1 inner hole, a pipe model with $57.6 \mathrm{M}$ boundary segments and 9 holes, and three coastal ocean/terrain regions : the Gulf of Mexico, Matagorda Bay, and West bay, with 230M, 250M, and 550M boundary vertices, respectively. The generated meshes of the two bays and the Gulf of Mexico have about 3, 4.5, and 10 billion elements, respectively.

We compare algorithms in three aspects: (1) Decomposition Quality: the workload balance ratio, total separator length, and separator angle deviation. (2) Parallel Computation Efficiency: the running time on each working processors and the total speedup in quad meshing. (3) Meshing Quality: the scaled-Jacobian of quad elements and number of singularities of the final mesh.

Our experiments are designed to compare 4 decomposition methods: (1) Partitioning via direct $L_{\infty}$-CVT, (2) Partitioning by METIS [20], a very widely used Graph Partitioning solver, (3) Partitioning by Medial Axis Domain Decomposition (MADD) [23], and (4) Our $L_{\infty}$-CVT-GP algorithm.

\subsection{Partitioning Quality Comparison}

Figure 8 illustrates the four partitioning results on the key and pipe models. Table 3 gives the partitioning statistics for key and pipe model respectively. The workload balance ratio $R_{W}$ is calculated following Eq. (2). When $R_{W}$ is closer to 1 , a better workload balance is achieved. The average compactness $\hat{R}_{C}$ is calculated following Eq. (5). The average separator angle deviation $\hat{\delta}_{\theta}$ is calculated following Eq. (8). Note that for each of these three terms, the smaller the measured value is, the better. From these experiments, we can see that:

1) For workload balance: METIS leads to the smallest $R_{W}$. The $R_{W}$ of our method is about $5 \%$ bigger than METIS. $R_{W}$ of MADD is about 


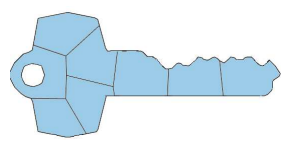

(a)

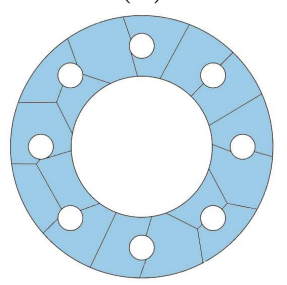

(e)

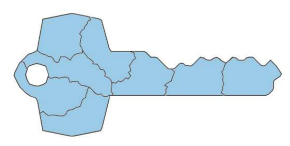

(b)

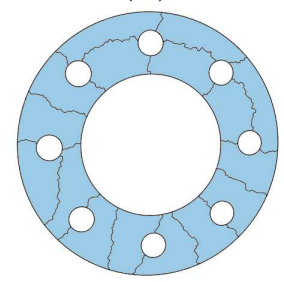

(f)

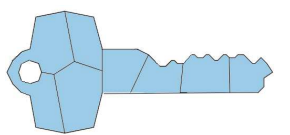

(c)

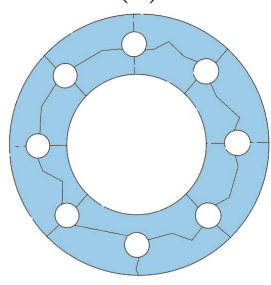

(g)

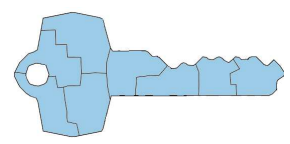

(d)

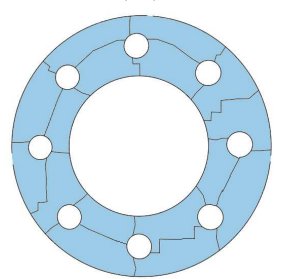

(h)

Figure 8: (a-d) Partitioning the key model into 8 subregions using direct $L_{\infty}$-CVT, METIS, MADD, and our $L_{\infty}$-CVT-GP algorithm. (e-h) Partitioning the pipe model into 16 subregions using direct $L_{\infty}$-CVT, METIS, MADD and our algorithm.

Table 3: Decomposition quality comparison (the key and pipe model): direct $L_{\infty}$-CVT, METIS, MADD and our $L_{\infty}$-CVT-GP method (initialized 4000 CVT Cells). $N_{S}$ is the number of subregions. Our method has comparable workload balance and average compactness, while our $\hat{\delta}_{\theta}$ is up to about $50 \%$ and $55 \%$ smaller than the METIS method on key and pipe model respectively; our $\hat{\delta}_{\theta}$ is $20 \%$ and $15 \%$ smaller than MADD on two models respectively. Compared with $L_{\infty}$ method, our $R_{W}$ is $40 \%$ smaller.

\begin{tabular}{|c|c|c|c|c|c|c|c|c|c|c|c|c|}
\hline Key & \multicolumn{3}{|c|}{$L_{\infty}$-CVT } & \multicolumn{3}{|c|}{ METIS } & \multicolumn{3}{|c|}{ MADD } & \multicolumn{3}{|c|}{ Our Method (4000 cells) } \\
\hline$N_{S}$ & 32 & 128 & 1024 & 32 & 128 & 1024 & 32 & 128 & 1024 & 32 & 128 & 1024 \\
\hline$R_{W}$ & 2.31 & 2.01 & 1.92 & 1.06 & 1.03 & 1.02 & 1.21 & 1.13 & 1.08 & 1.08 & 1.06 & 1.04 \\
\hline$\hat{R}_{C}$ & 3.93 & 13.25 & 104.3 & 3.61 & 12.21 & 93.31 & 3.98 & 14.26 & 104.91 & 3.85 & 13.57 & 105.21 \\
\hline$\hat{\delta}_{\theta}$ & $0.21 / 0.14$ & $0.18 / 0.15$ & $0.15 / 0.14$ & $0.40 / 0.24$ & $0.32 / 0.26$ & $0.41 / 0.21$ & $0.36 / 0.16$ & $0.32 / 0.20$ & $0.25 / 0.19$ & $0.16 / 0.14$ & $0.17 / 0.15$ & $0.15 / 0.14$ \\
\hline Pipe & & $L_{\infty}$-CVT & & & METIS & & & MADD & & Our & ethod $(400$ & cells) \\
\hline$N_{S}$ & 32 & 128 & 1024 & 32 & 128 & 1024 & 32 & 128 & 1024 & 32 & 128 & 1024 \\
\hline$R_{W}$ & 2.39 & 2.09 & 1.98 & 1.07 & 1.05 & 1.04 & 1.27 & 1.25 & 1.13 & 1.14 & 1.13 & 1.10 \\
\hline$\hat{R}_{C}$ & 1.34 & 4.76 & 36.51 & 1.18 & 4.64 & 36.37 & 1.32 & 5.06 & 35.48 & 1.25 & 4.71 & 36.42 \\
\hline$\hat{\delta}_{\theta}$ & $0.22 / 0.14$ & $0.18 / 0.15$ & $0.15 / 0.15$ & $0.41 / 0.23$ & $0.32 / 0.25$ & $0.42 / 0.21$ & $0.36 / 0.15$ & $0.32 / 0.20$ & $0.25 / 0.21$ & $0.18 / 0.12$ & $0.15 / 0.12$ & $0.13 / 0.11$ \\
\hline
\end{tabular}

$10 \%$ bigger than ours. The direct CVT partitioning has worst workload balance and its $R_{W}$ is about $55 \%$ bigger than ours.

2) For average compactness $\hat{R}_{C}$ : METIS also leads to the most compact subregions. The $\hat{R}_{C}$ of our method is about $8 \%$ bigger than METIS, but $6 \%$ smaller than MADD, and about $2 \%$ smaller than direct CVT partitioning.

3) For average separator angle deviation $\hat{\delta}_{\theta}$ : our algorithm has the smallest separator angle deviation. Our $\hat{\delta}_{\theta}$ is $40 \%$ smaller than METIS, $20 \%$ smaller than MADD, and about $5 \%$ smaller than direct CVT partitioning. In Conclusion, our algorithm results in significantly smaller separator an- 


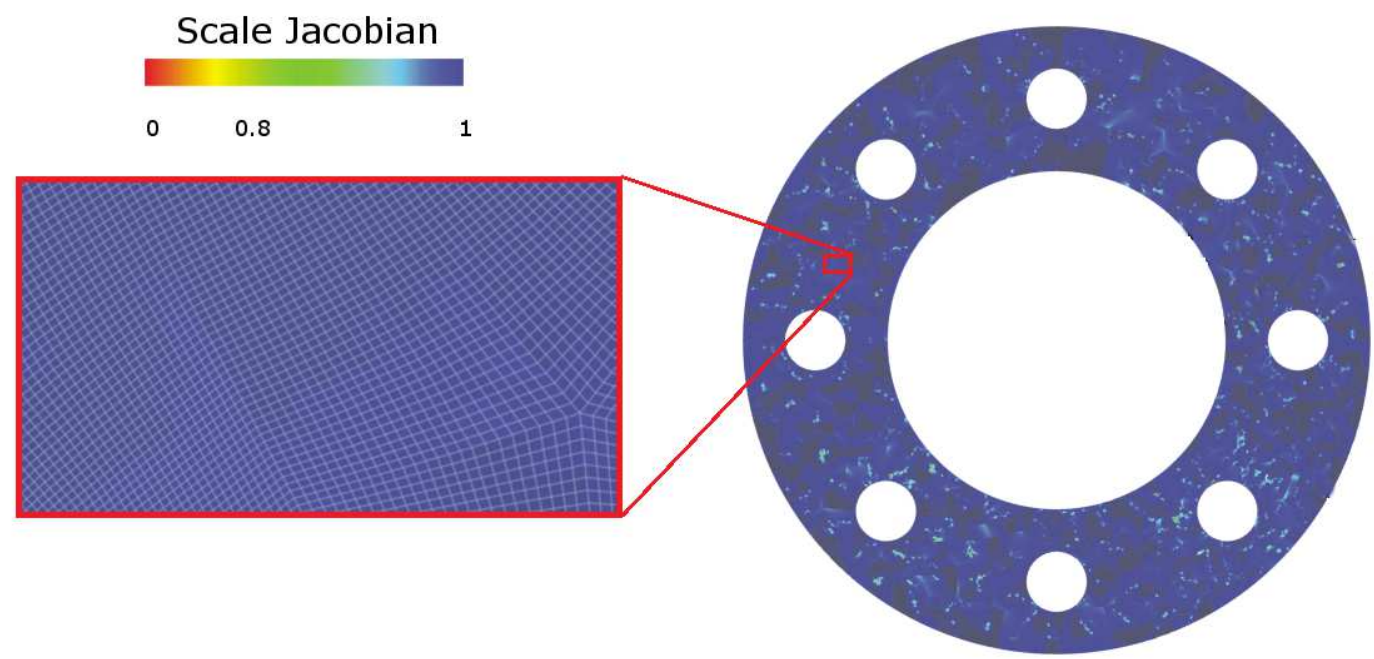

Figure 9: Quad meshing result of the Pipe model, color-coded by elements' scaled Jacobian values. The color from blue to red indicates the mesh quality from high to low.

gle deviation than the METIS and MADD method, while preserving good workload balance and compactness. Compared with the direct CVT decomposition, our algorithm yields $40 \%$ smaller workload balance ratio, while have slightly better the compactness and the separator angle deviation small. This indicates that meshing based on our decomposition is about $20 \%$ faster than that using CVT decomposition. To achieve similar workload balance, the CVT method needs to use much more (in our experiments, more than 4 times) cells. Then as a side effect, this will lead to a significant increase in $L_{S}$ and in singularities of the final quad mesh (see Section 5.3), which is undesirable.

\subsection{Parallel Computational Efficiency}

Sub-regions are distributed to different working processors for simultaneous quad mesh generation using advancing front. We test the parallel computation efficiency on our datasets: the pipe model (Figure 9), the terrain near West Bay (Figure 10(a)), and the terrain near Matagorda Bay (Figure 10(b)) and the entire ocean region of the Gulf of Mexico (Figure 10(c)). Figure 11(a) shows the actual meshing time on each working processor. The working time on different processors are very balanced during the parallel execution. 


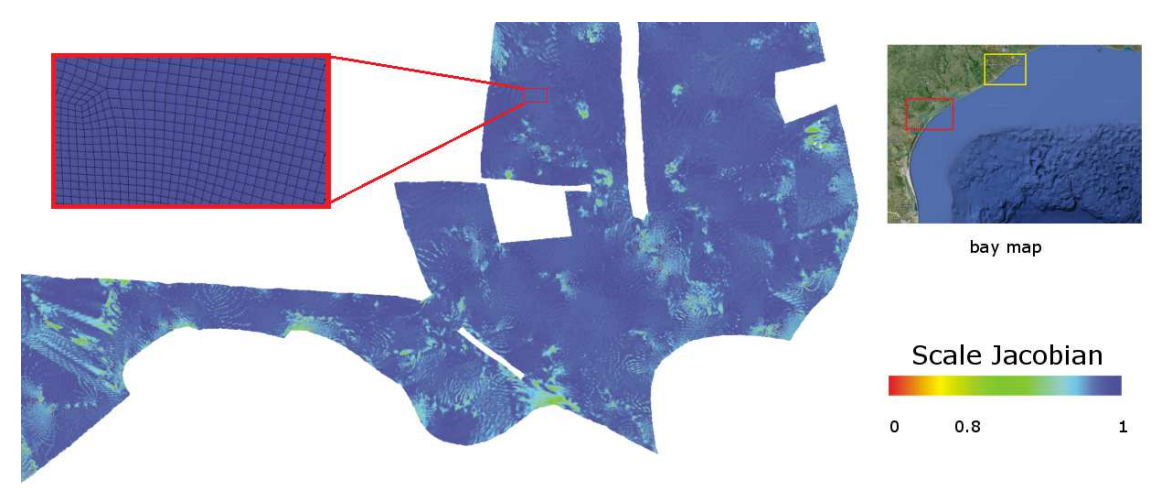

(a)

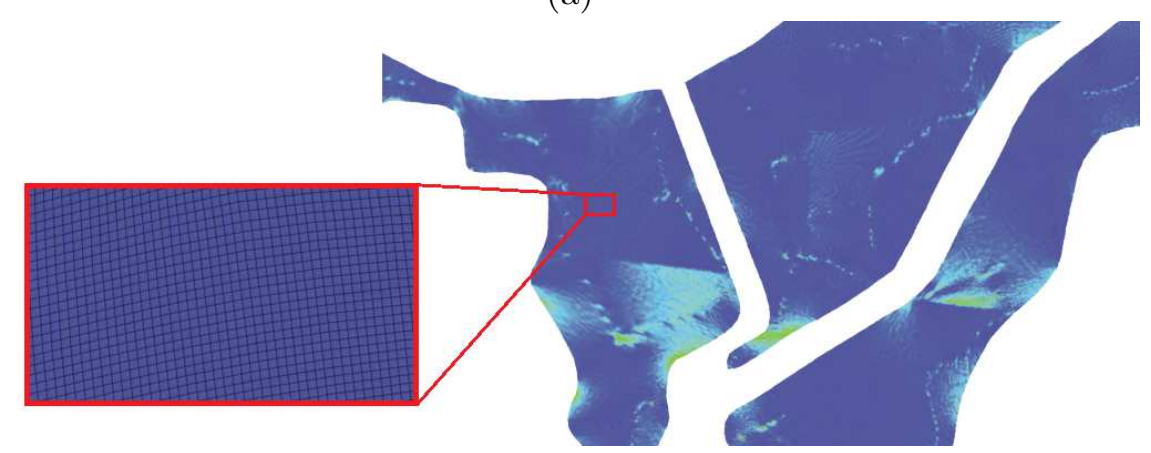

(b)
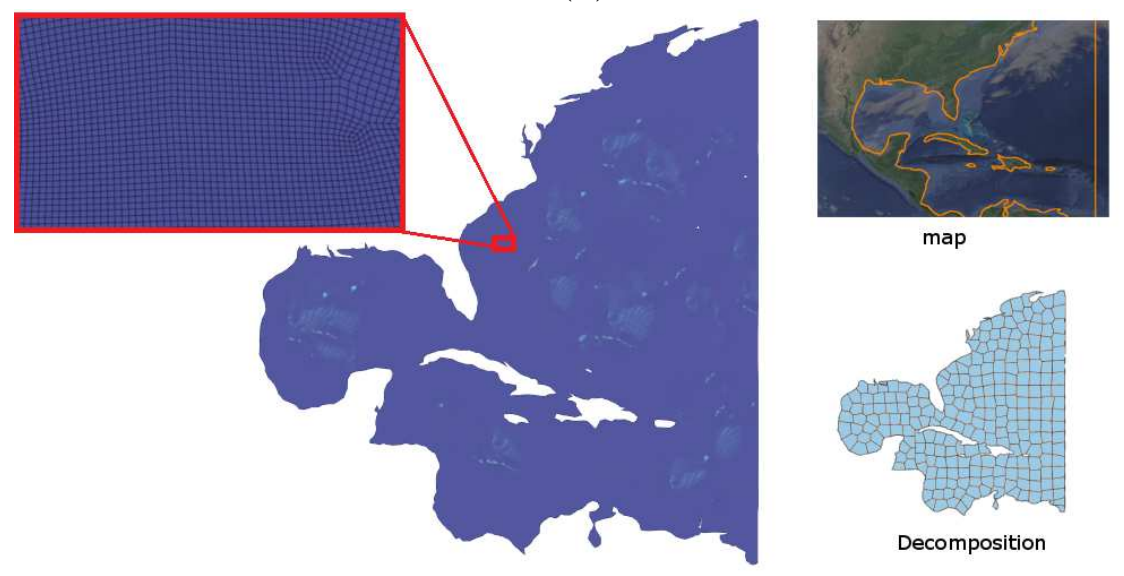

map

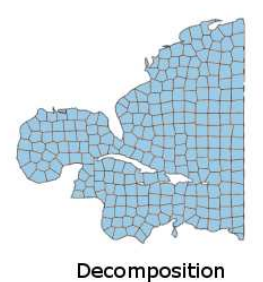

(c)

Figure 10: (a, b, c) The quad meshes for West Bay (yellow region in bay map), Matagorda Bay (red region in bay map), and the Gulf of Mexico respectively. The quad meshes are color-coded in element's scaled Jacobian value. 
Table 4: The Runtime Table for different partitioning algorithms. $N_{S}$ is the number of subregions. The runtime is in second, and includes the partitioning, meshing and relaxation time. Usually, the METIS is fastest, and our method has the second best efficiency.

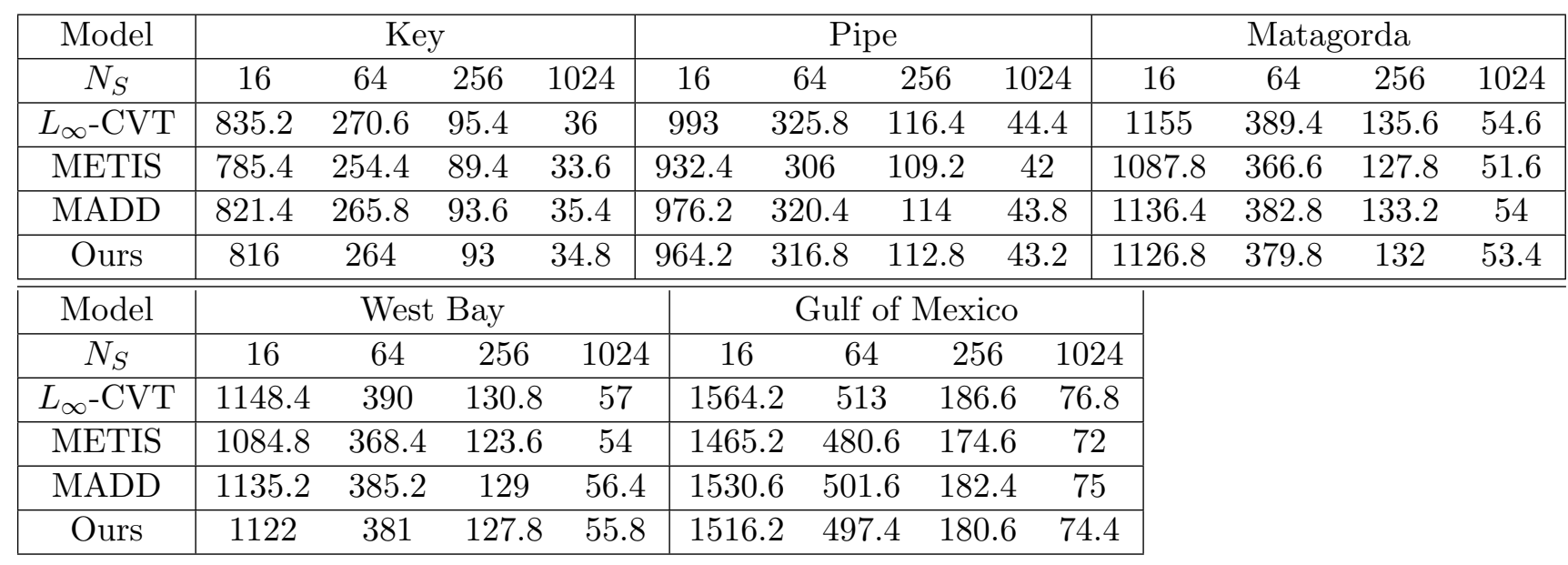

We also run the experiments by changing the number of working processors. The total run time is shown in Table 4. METIS leads the fastest total running time, Our algorithm is about $7 \%$ slower than METIS, $1 \%$ and $3 \%$ faster than MADD and direct CVT respectively. Ideally, when the computation is evenly distributed to each processor, the speed up would be $T / D$, where $T$ is the total time cost of our algorithm without parallelization, and $D$ is the number of processors. But there are overheads of this divide-andconquer pipeline, including the partitioning, data transmission, and postprocessing. The workload balancing also affects the speed-up performance. Figure 11 (b) plots the speedup of our algorithm on different models. The yellow dot line is the ideal speedup, and our algorithm's parallel speed up ranges from 2.78 to 601.5 when 4 to 1024 processors are used, respectively. The partitioning time usually takes up to $30 \%$ of the total time. To improve the meshing quality near the separator, we apply a Laplacian relaxation [37] near the separators after individually generated meshes are merged. Hence, a smaller $L_{S}$ will reduce this post-processing time. In our experiments, the relaxation takes about $4 \%$ of the total computation time.

\subsection{Meshing Quality Comparison}

Table 5 compares the quality of final quad meshes generated by the sequential algorithm and four parallel algorithms using different partitioning 


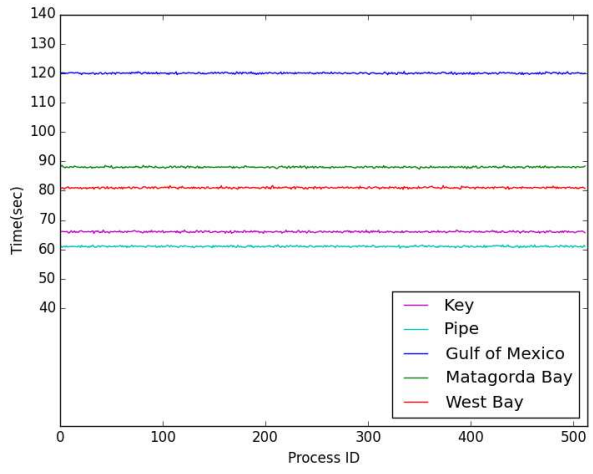

(a)

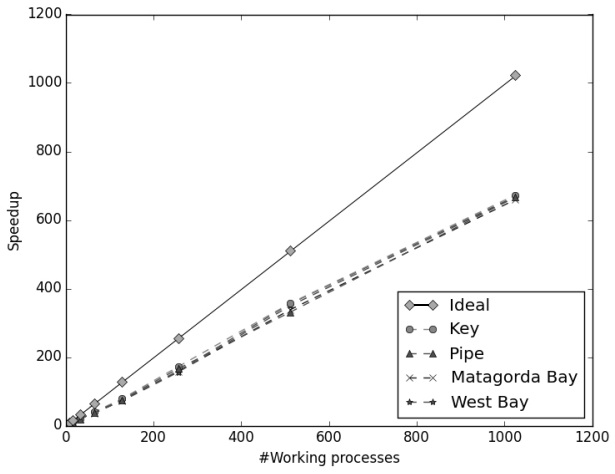

(b)

Figure 11: (a) The load balance for 512 working processes of the meshing of our dataset. (b) The parallel speedup of meshing: we test our algorithm using 4 to 1024 working processes. The black solid line is the ideal speedup, and the speed up of our algorithm on different models ranges from 2.78 to 601.5 .

\begin{tabular}{|c|c|c|c|}
\hline Model $\left(N_{S}\right)$ & Sequential Algorithm & $L_{\infty}$-CVT & METIS \\
\hline Key $(32)$ & $0.97 / 0.13 / 0.39 / 35$ & $0.98 / 0.14 / 0.38 / 233$ & $0.93 / 0.27 / 0.23 / 411$ \\
\hline Pipe (16) & $0.97 / 0.04 / 0.31 / 34$ & $0.97 / 0.04 / 0.30 / 55$ & $0.94 / 0.09 / 0.23 / 128$ \\
\hline Matagorda Bay $(512)$ & - & $0.97 / 0.04 / 0.35 / 325$ & $0.93 / 0.16 / 0.21 / 413$ \\
\hline West Bay $(512)$ & - & $0.96 / 0.05 / 0.36 / 235$ & $0.93 / 0.12 / 0.24 / 562$ \\
\hline Gulf of Mexico $(1024)$ & - & $0.97 / 0.04 / 0.36 / 3158$ & $0.96 / 0.15 / 0.21 / 3491$ \\
\hline \hline Model $\left(N_{S}\right)$ & MADD & Our Method \\
\hline Key $(32)$ & $0.97 / 0.18 / 0.23 / 226$ & $0.98 / 0.13 / 0.38 / 216$ \\
\hline Pipe (16) & $0.95 / 0.07 / 0.23 / 84$ & $0.97 / 0.04 / 0.30 / 50$ \\
\hline Matagorda Bay (512) & $0.96 / 0.08 / 0.27 / 346$ & $0.97 / 0.04 / 0.35 / 305$ \\
\hline West Bay (512) & $0.95 / 0.07 / 0.28 / 321$ & $0.98 / 0.05 / 0.36 / 225$ \\
\hline Gulf of Mexico $(1024)$ & $0.96 / 0.09 / 0.23 / 3201$ & $0.98 / 0.04 / 0.36 / 3104$ \\
\hline
\end{tabular}

Table 5: Mesh Quality Comparison between the sequential meshing algorithm, $L_{\infty}$-CVT, METIS, MADD, and our algorithm. The sequential algorithm applies the advancing front without partitioning; and it only works on small models such as the Key and Pipe. $N_{S}$ is the number of subregions. The four values: $\kappa_{a v g}, \sigma_{\kappa}, \kappa_{m i n}, \# S$, namely, (1) average, (2) standard deviation, and (3) minimum of the scaled Jacobian, and (4) the number of singularities, are listed to show meshing quality. The scaled Jacobian of our mesh is comparable to the sequential algorithm, but our mesh, like other meshes generated through region partitioning, has significantly more singularities than the mesh generated by sequential meshing. The average and minimal scaled Jacobians of our algorithm are $10 \%$ and $4 \%$ better than METIS and MADD, respectively. Our singularities are $40 \%$ and $20 \%$ fewer than METIS and MADD. Compared with $L_{\infty}$-CVT, our algorithm leads to about $4 \%$ better average and minimal scaled Jacobians and $8 \%$ fewer singularities. 
methods. The sequential algorithm applies the advancing front to generate quad mesh without partitioning. For large model such as Matagorda Bay, West Bay and Gulf of Mexico the sequential algorithm failed to get a result. For each quad mesh, we compute four values, $\kappa_{a v g}, \sigma_{\kappa}, \kappa_{m i n}, \# S$, namely, the (1) average, (2) standard deviation, and (3) minimum of scaled Jacobian, and (4) the number of singularities. Ideally, the scaled Jacobian for every element should be 1 . The scaled Jacobian of our mesh is comparable to the sequential algorithm. But due region partitioning and individual advancing front mesh generation, our mesh have more singularities. Compared with other partitioning techniques, our average and minimal scaled Jacobians are $10 \%$ and $4 \%$ better than METIS and MADD respectively. Our singularities are $40 \%$ and $20 \%$ fewer than METIS and MADD. Compared with $L_{\infty}$ CVT, our algorithm leads to about $4 \%$ better average and minimal scaled Jacobians and $8 \%$ fewer singularities. These experiments show that our algorithm produces higher-quality quad meshes than other partitioning algorithms.

\section{Simulation Experiments}

To evaluate how tessellation quality affects the numerical simulations that run on the mesh, we perform experiments that solve partial differential equations in two common example engineering analysis of mechanical systems: (1) Heat Diffusion Simulation and (2) Large-Eddy Simulation. The first simulation solves a heat equation that is being widely used in many areas such as economics, image processing, bioengineering, etc. [38]. The second simulation is on turbulence that is also widely applied in a variety of engineering applications such as combustion, acoustics, and simulation of atmospheric boundary layer [39].

Comparing Simulation Efficiency: Convergence of Error Residual. We examine the error residual on four types of meshes: (1) triangle meshes, (2) quad mesh generated by METIS, (3) quad mesh generated by MADD decomposition, (4) quad mesh generated by our $L_{p}$-CVT decomposition. The simulation environment is a workstation with $2.27 \mathrm{GHz} \mathrm{CPU}$ and 8 GB memory. The software is OpenFOAM[40], an open source objectoriented library for numerical simulations in continuum mechanics written in $\mathrm{C}++$.

Comparing Simulation Accuracy: Simulation Results versus Analytical Solution. We also compare the simulated results with analytical solution to see whether/how different meshes affect the simulation accuracy. 


\subsection{Heat Diffusion Simulation}

The heat diffusion simulation solves the Laplacian Equation:

$$
\frac{\partial T}{\partial t}+\nabla\left(D_{t} \nabla T\right)=0
$$

where $T$ is the temperature and coefficient $D_{t}$ is the thermal conductivity.

We run the simulation on the key domain (Fig. 4(a)), initialize with boundary temperature as $273 K$ (Kelvin) and interior as $0 K$. The boundary condition is a fixed $273 \mathrm{~K}$. The Fig. 12 shows the iteration-residual plot. The statistics are shown in Table. 6. From the table we can see that: (1) The error residuals from the simulations performed on the quad meshes are one to two order of magnitude smaller than that on the triangle meshes. (2) Among the three quad meshes examined in this paper, the mesh generated by our proposed $L_{p}$-CVT-based algorithm also produces significantly smaller error residuals in simulation.

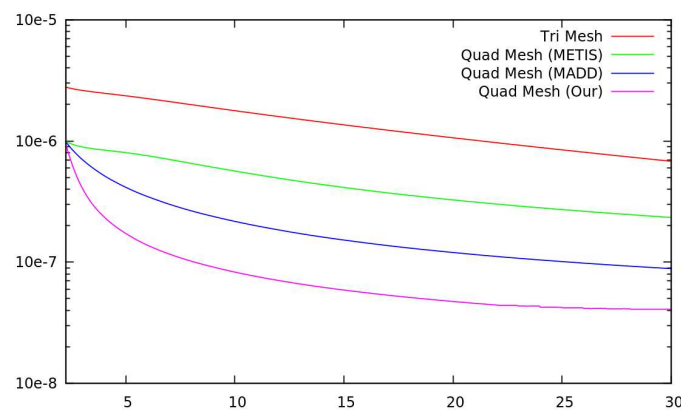

(a)

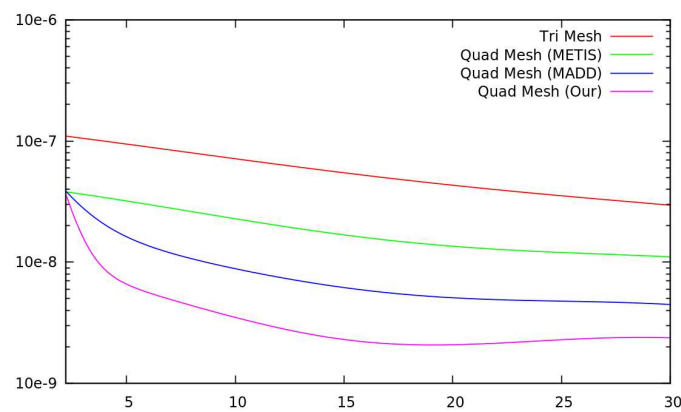

(b)

Figure 12: The iteration-residual plot of solving a Laplacian on four meshes, with different resolutions. (a) $9.9 \mathrm{~K}$ elements resolution meshes. (b) 40k elements resolution meshes.

\subsection{Large-Eddy Simulation}

Computing and modeling turbulent flows has many applications in engineering area. The principal difficulty is the dominance of non-linear effects as well as the continuous and wide spectrum of observed scales. In practical applications, direct numerical simulations of the turbulent flows are computational expensive and often prohibitive [41]. Large-Eddy Simulation uses a subgrid-scale model to balance the computational efficiency and the result accuracy [42]. The governing equations are: 
Table 6: Simulation statistics on meshes including triangle mesh, quad meshes generated by METIS, MADD and our $L_{\infty}$-CVT-GP algorithms.

\begin{tabular}{|c|c|c|c|c|}
\hline & \# Element & \# Singularity & Min. Scaled Jacobian & Residual \\
\hline Triangle Mesh & $9.8 \mathrm{k}$ & - & - & $4.0 \times 10^{-6}$ \\
\hline METIS & $9.8 \mathrm{k}$ & 485 & 0.24 & $2.3 \times 10^{-7}$ \\
\hline MADD & $9.8 \mathrm{k}$ & 308 & 0.28 & $8.8 \times 10^{-8}$ \\
\hline$L_{\infty}$-CVT-GP & $9.9 \mathrm{k}$ & 203 & 0.36 & $1.5 \times 10^{-8}$ \\
\hline Triangle Mesh & $40 \mathrm{k}$ & - & - & $2.9 \times 10^{-8}$ \\
\hline METIS & $40 \mathrm{k}$ & 2043 & 0.25 & $1.1 \times 10^{-8}$ \\
\hline MADD & $40 \mathrm{k}$ & 1504 & 0.28 & $4.4 \times 10^{-9}$ \\
\hline$L_{\infty}$-CVT-GP & $40 \mathrm{k}$ & 1044 & 0.35 & $2.3 \times 10^{-9}$ \\
\hline
\end{tabular}

$$
\left[\begin{array}{c}
\rho \\
\rho u \\
\rho v \\
E
\end{array}\right]+\left[\begin{array}{c}
\rho u \\
\rho u^{2}+p \\
\rho u v \\
u(E+p)
\end{array}\right]+\left[\begin{array}{c}
\rho v \\
\rho u v \\
\rho v^{2}+p \\
v(E+p)
\end{array}\right]=0
$$

where $\rho$ is the density of compressible fluids around the airfoil, $u$ and $v$ are the tangential velocity and the normal velocity field of the fluid respectively. $p$ is the pressure field of the fluid. $E$ is the total energy density (Energy in unit volume):

$$
E=\frac{1}{2} \rho u^{2}+\rho c_{v} \frac{p}{\rho R}
$$

where $c_{v}$ is the specific heat a constant volume, $R$ is the special gas constant.

The airfoil domain in this simulation is shown in Fig. 14 (a). (b) - (d) are the decomposition results generated by METIS, MADD and our $L_{p}$ CVT, respectively. The iteration-residual plot on different meshes are shown in Fig. 13. From the statistics Table 7 we can see that: (1) The error residuals from the simulations performed on the quad meshes are almost one to two order of magnitude smaller than that on the triangle meshes. (2) The mesh generated by our proposed $L_{p}$-CVT-based algorithm leads to $50 \%$ to $75 \%$ smaller error residual.

\subsection{Simulation Accuracy}

To evaluate how mesh quality affects the simulation accuracy. We conducted another heat diffusion experiment, where an analytical solution can be 


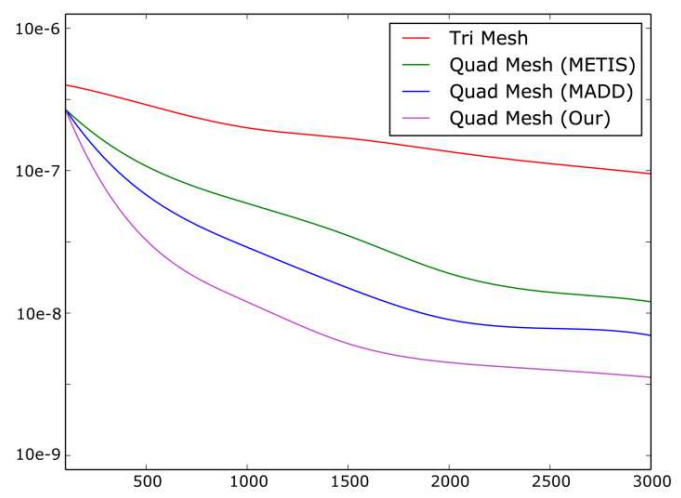

Figure 13: The iteration-residual plot of the Large-Eddy simulation on four meshes.

\section{Domain}

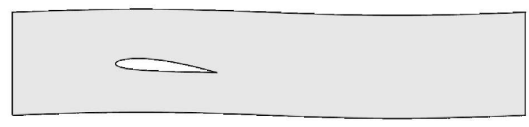

\section{MADD}

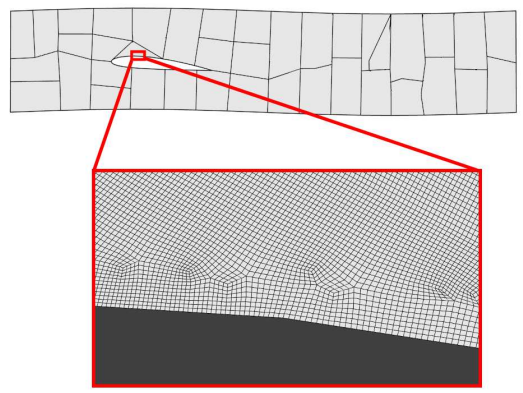

\section{METIS}

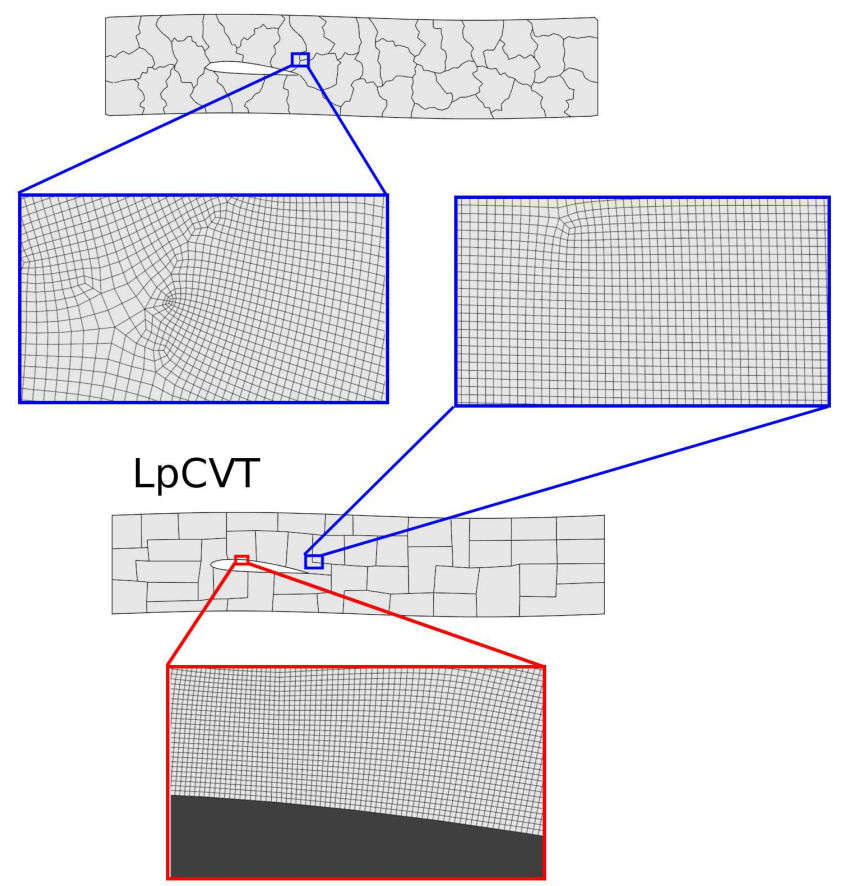

Figure 14: The airfoil domain and three different decomposition results. Zoom-ins are the final mesh generated after the decomposition. 
Table 7: Statistics for airfoil simulation on triangle mesh, quad meshes generated by METIS, MADD and our $L_{\infty}$-CVT-GP algorithms.

\begin{tabular}{|c|c|c|c|c|}
\hline & \# Element & \# Singularity & Min. Scaled Jacobian & Residual \\
\hline Triangle Mesh & $220 \mathrm{k}$ & - & - & $9.5 \times 10^{-8}$ \\
\hline METIS & $220 \mathrm{k}$ & 1938 & 0.12 & $1.2 \times 10^{-8}$ \\
\hline MADD & $220 \mathrm{k}$ & 1304 & 0.17 & $6.9 \times 10^{-9}$ \\
\hline$L_{\infty}$-CVT-GP & $220 \mathrm{k}$ & 872 & 0.28 & $3.4 \times 10^{-9}$ \\
\hline
\end{tabular}

derived and used as the ground truth. Then, we can calculate the deviation between the simulation and analytical solutions.

The simulation domain $D$ is a two-dimensional disk of radius $a$ and the goal is to solve the temperature distribution $T(r, t)$ at position $r$ at time $t$. This disk has the initial temperature $T(r, 0)=0$ and the boundary condition $T(a, t)=1$. The analytical solution [43] is:

$$
T(r, t)=1-2 \sum_{n=1}^{\infty} \frac{J_{0}\left(\frac{\alpha_{n} r}{a}\right)}{\alpha_{n} J_{1}\left(\alpha_{n}\right)} e^{-\alpha_{n}^{2} \kappa t / a^{2}},
$$

where $\alpha_{n}$ is the $n$-th positive zero of the Bessel function of the first kind $J_{0}(x)[44], \kappa$ is the thermal diffusivity (we set $\kappa=1$ in our problem).

Then we run the simulation on this domain in OpenFoam, to obtain the temperature distribution at different time $t$, and measure the deviation between the simulation and analytical results after the simulations converge.

This deviation $\epsilon$ (that can be considered as a measure of simulation error) at time $t$ is measured by

$$
\epsilon_{t}=\max _{v_{i} \in V(D)}\left\|T_{G}\left(v_{i}, t\right)-T_{S}\left(v_{i}, t\right)\right\|
$$

where $V(D)$ is the vertex set of the mesh of domain $D, T_{G}(v, t)$ and $T_{S}(v, t)$ are the analytical and simulated temperature values at vertex $v$, respectively. We illustrate the simulation errors on different meshes, by $\epsilon$, at $t=1, t=5$ and $t=10$ in Table 8 and Figure 15. From the experiments, we can observe that: (1) $\epsilon$ obtained on the quad meshes are smaller than that on the triangle mesh. (2) The mesh generated by our proposed $L_{p}$-CVT-based algorithm outperforms other quad meshes in terms of the simulation accuracy, measured by $\epsilon$. Our mesh yields smaller simulation $\epsilon$ than other quad meshes. 
Table 8: Simulation Error $\epsilon$ (Equation (16)) of Heat diffusion simulations on Delaunay triangle mesh and quadrilateral meshes generated by advancing front using METIS, MADD, and our $L_{\infty}$-CVT-GP partitioning algorithms.

\begin{tabular}{|c|c|c|c|c|}
\hline & \# Element & $\epsilon(t=1)$ & $\epsilon(t=5)$ & $\epsilon(t=10)$ \\
\hline Triangle Mesh & $10 \mathrm{k}$ & $3.7 \times 10^{-2}$ & $1.6 \times 10^{-2}$ & $1.1 \times 10^{-2}$ \\
\hline METIS & $10 \mathrm{k}$ & $1.9 \times 10^{-2}$ & $1.0 \times 10^{-2}$ & $0.8 \times 10^{-2}$ \\
\hline MADD & $10 \mathrm{k}$ & $1.2 \times 10^{-2}$ & $0.9 \times 10^{-2}$ & $0.6 \times 10^{-2}$ \\
\hline$L_{\infty}$-CVT-GP & $10 \mathrm{k}$ & $0.8 \times 10^{-2}$ & $0.7 \times 10^{-2}$ & $0.5 \times 10^{-2}$ \\
\hline
\end{tabular}

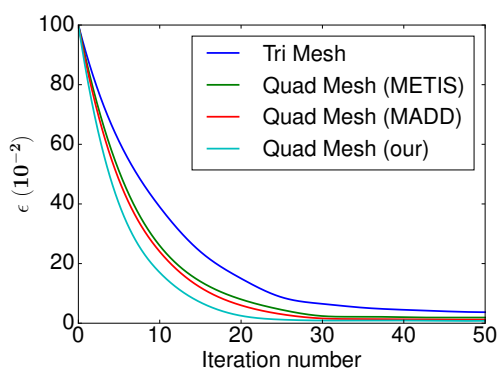

(a) $\epsilon(t=1)$

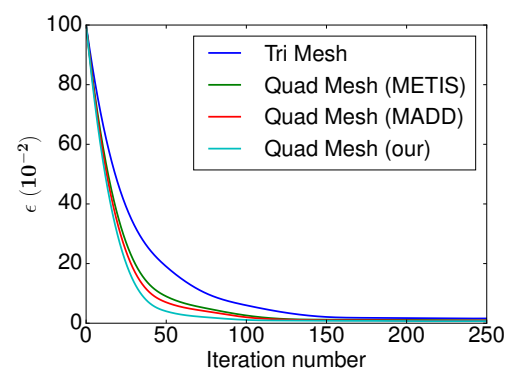

(b) $\epsilon(t=5)$

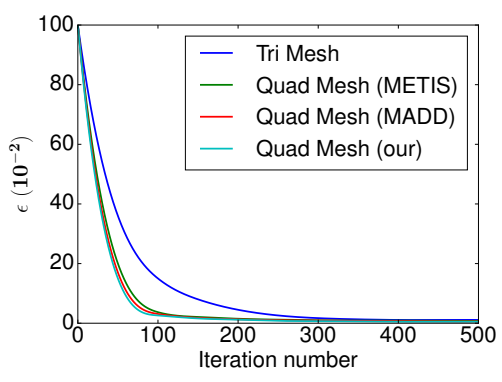

(c) $\epsilon(t=10)$

Figure 15: Simulation error $\epsilon$ of heat diffusion on four meshes, at different time $t$.

\section{Conclusions}

We present a parallel quad mesh generation pipeline for large-scale 2D geometric regions. A main contribution of this work is the solving of data partitioning with effective incorporation of the geometric constraint on angles between separators. After partitioning, subregions are distributed to different processors for parallel mesh generation through advancing front. Finally, after composition, post-processing is performed near partitioning boundaries for refinement. We evaluate our partitioning and mesh generation algorithm in different experiments. Compared with other data partitioning strategies, our new algorithm leads to better partition result and final meshing quality.

We set up a project website and a demo server for general users to upload or interactively sketch a $2 \mathrm{D}$ region (boundary) to run our partitioning and meshing algorithm. This provides an interactive environment to demonstrate our algorithm. We also plan to integrate this server and its execution with the LSU HPC nodes. Tasks submitted by the user will be computed in clusters, the results will be temporally stored for the user to download. This server is 
https://gvc.cct.lsu.edu/LargeScaleMeshing.

In the future, we will generalize this parallel pipeline for structured meshing of curved 2D manifolds and 3D solid regions. We will also investigate parallel meshing algorithms with controlled singularity numbers and distributions. For this purpose, singularity estimation needs to be incorporated in partition optimization; tessellation of subregions may be computed through parameterization-based mesh construction algorithms [45, 46] which allows us to globally control and optimize the singularity distribution. Post-processing algorithms that allows adaptively merging of nearby singularities may also be useful. Another modification to the model can be to directly formulate the partitioning as a $k$-way partition, rather than recursive 2 -way partitions. Although this will not fundamentally change the complexity of the model, in practice, it could result in more balanced partitioning when the processor numbers are not powers of 2 . Finally, we will also explore more efficient computation of geometric-aware graph partitioning using better mathematical models and solving schemes, such as block exchange generalization of the Kernighan and Lin algorithm [47], multilevel local improvement [48], or appropriate regularization [49].

\section{Acknowledgements}

This research is supported by NSF IIS-1320959, IIS-1251095, and CNS1205682. We also thank reviewers for their valuable suggestions and comments.

[1] A. Cifuentes, A. Kalbag, A performance study of tetrahedral and hexahedral elements in 3-d finite element structural analysis, Finite Elements in Analysis and Design 12 (3-4) (1992) 313 - 318.

[2] L. Linardakis, N. Chrisochoides, Delaunay decoupling method for parallel guaranteed quality planar mesh refinement, SIAM JSC 27 (4) (2006) $1394-1423$.

[3] R. Löhner, A 2nd generation parallel advancing front grid generator, in: Proc. Int. Meshing Roundtable, 2013, pp. 457-474.

[4] T. Panitanarak, S. M. Shontz, Mdec: Metis-based domain decomposition for parallel $2 \mathrm{~d}$ mesh generation, Procedia Computer Science 4 (2011) 302-311. 
[5] D. Nave, N. Chrisochoides, L. P. Chew, Guaranteed: quality parallel delaunay refinement for restricted polyhedral domains, in: Proceedings of the eighteenth annual symposium on Computational geometry, 2002, pp. 135-144.

[6] Y. Ito, A. M. Shih, A. K. Erukala, B. K. Soni, A. Chernikov, N. P. Chrisochoides, K. Nakahashi, Parallel unstructured mesh generation by an advancing front method, Mathematics and Computers in Simulation 75 (5) (2007) 200-209.

[7] A. Shamir, A survey on mesh segmentation techniques, in: Computer graphics forum, Vol. 27, 2008, pp. 1539-1556.

[8] A. Agathos, I. Pratikakis, S. Perantonis, N. Sapidis, P. Azariadis, 3d mesh segmentation methodologies for cad applications, Computer-Aided Design 4 (6) (2007) 827-841.

[9] X. Chen, A. Golovinskiy, T. Funkhouser, A benchmark for 3D mesh segmentation, ACM TOG. 28 (3) (2009) 1-12.

[10] M. O. Freitas, P. A. Wawrzynek, J. B. Cavalcante-Neto, C. A. Vidal, L. F. Martha, A. R. Ingraffea, A distributed-memory parallel technique for two-dimensional mesh generation for arbitrary domains, Adv. Eng. Softw. 59 (2013) 38-52.

[11] J. Gould, D. Martineau, R. Fairey, Automated two-dimensional multiblock meshing using the medial object, in: Proc. of the 20th Int'l Meshing Roundtable, Springer, 2012, pp. 437-452.

[12] K. Brix, S. S. Melian, S. Müller, G. Schieffer, Parallelisation of multiscale-based grid adaptation using space-filling curves, in: ESAIM: Proceedings, Vol. 29, EDP Sciences, 2009, pp. 108-129.

[13] M. F. Mokbel, W. G. Aref, Irregularity in high-dimensional space-filling curves, Distributed and Parallel Databases 29 (3) (2011) 217-238.

[14] P. MacNeice, K. M. Olson, C. Mobarry, R. de Fainchtein, C. Packer, Paramesh: A parallel adaptive mesh refinement community toolkit, Computer physics communications 126 (3) (2000) 330-354. 
[15] H. Ji, F.-S. Lien, E. Yee, A new adaptive mesh refinement data structure with an application to detonation, Journal of Computational Physics 229 (23) (2010) 8981-8993.

[16] A. Maximo, L. Velho, M. Siqueira, Adaptive multi-chart and multiresolution mesh representation, Computers \& Graphics 38 (2014) 332-340.

[17] M. Attene, M. Mortara, M. Spagnuolo, B. Falcidieno, Hierarchical convex approximation of $3 \mathrm{~d}$ shapes for fast region selection, in: CGF, Vol. 27, 2008, pp. 1323-1332.

[18] B. W. Kernighan, S. Lin, An efficient heuristic procedure for partitioning graphs, Bell system technical journal 49 (2) (1970) 291-307.

[19] A. Pothen, H. D. Simon, K.-P. Liou, Partitioning sparse matrices with eigenvectors of graphs, SIAM Journal on Matrix Analysis and Applications 11 (3) (1990) 430-452.

[20] G. Karypis, V. Kumar, A fast and high quality multilevel scheme for partitioning irregular graphs, SIAM JSC 20 (1) (1998) 359-392.

[21] E. Vecharynski, Y. Saad, M. Sosonkina, Graph partitioning using matrix values for preconditioning symmetric positive definite systems, Journal on Sci. Computing 36 (1) (2014) A63-A87.

[22] H. Meyerhenke, Dynamic load balancing for parallel numerical simulations based on repartitioning with disturbed diffusion, in: Parallel and Distributed Systems (ICPADS), 2009 15th International Conference on, IEEE, 2009, pp. 150-157.

[23] L. Linardakis, N. Chrisochoides, A static medial axis domain decomposition for 2d geometries, ACM TOMS 34 (1) (2005) 1-19.

[24] S. OWEN, M. STATEN, S. CANANN, S. SAIGAL, Q-morph: An indirect approach to advancing front quad meshing, Int. J. Numer. Meth. Engng 44 (1999) 1317-1340.

[25] J.-F. Remacle, F. Hernrotte, T. Carrier-Baudouin, E. Béchet, E. Marchandise, C. Geuzaine, T. Mouton, A frontal delaunay quad mesh generator using the $l^{\infty}$ norm, International Journal for Numerical Methods in Engineering (2010) 1-6. 
[26] X. Liang, M. S. Ebeida, Y. Zhang, Guaranteed-quality all-quadrilateral mesh generation with feature preservation, Computer Methods in Applied Mechanics and Engineering 199 (29) (2010) 2072-2083.

[27] S.-W. Chae, J.-H. Jeong, Unstructured surface meshing using operators, in: Proc. Int. Meshing Roundtable, 1997, pp. 281-291.

[28] T. D. Blacker, M. B. Stephenson, Paving: A new approach to automated quadrilateral mesh generation, International Journal for Numerical Methods in Engineering 32 (4) (1991) 811-847.

[29] D. Bommes, B. Levy, N. Pietroni, E. Puppo, C. Silva, M. Tarini, D. Zorin, Quad-mesh generation and processing: A survey, CGF 32 (6) (2013) 51-76.

[30] F. Kälberer, M. Nieser, K. Polthier, Quadcover-surface parameterization using branched coverings, in: CGF, Vol. 26, 2007, pp. 375-384.

[31] J.-F. Remacle, F. Henrotte, T. C. Baudouin, C. Geuzaine, E. Béchet, T. Mouton, E. Marchandise, A frontal delaunay quad mesh generator using the 1 norm, in: Proceedings of the 20th International Meshing Roundtable, Springer, 2012, pp. 455-472.

[32] M. Campen, L. Kobbelt, Quad layout embedding via aligned parameterization, in: CGF, Vol. 33, 2014, pp. 69-81.

[33] P. Bonami, L. T. Biegler, A. R. Conn, G. Cornuéjols, I. E. Grossmann, C. D. Laird, J. Lee, A. Lodi, F. Margot, N. Sawaya, et al., An algorithmic framework for convex mixed integer nonlinear programs, Discrete Optimization 5 (2) (2008) 186-204.

[34] B. Lévy, Y. Liu, Lp centroidal voronoi tessellation and its applications, ACM Trans. Graph. 29 (4) (2010) 119:1-119:11.

[35] Y. Liu, W. Wang, B. Lévy, F. Sun, D.-M. Yan, L. Lu, C. Yang, On centroidal voronoi tessellationenergy smoothness and fast computation, ACM Trans. on Graphics 28 (4) (2009) 101.

[36] S. Fortune, A sweepline algorithm for voronoi diagrams, Algorithmica 2 (1-4) (1987) 153-174. 
[37] W. Buell, B. Bush, Mesh generationa survey, Journal of Manufacturing Science and Engineering 95 (1) (1973) 332-338.

[38] G. Strang, K. Aarikka, Introduction to applied mathematics, Vol. 16, Wellesley-Cambridge Press Wellesley, MA, 1986.

[39] C. Moeng, P. Sullivan, Large eddy simulation, Encyclopedia of Atmospheric Sciences 1140 (2002) 1150.

[40] H. Jasak, A. Jemcov, Z. Tukovic, Openfoam: A c++ library for complex physics simulations, in: International workshop on coupled methods in numerical dynamics, Vol. 1000, 2007, pp. 1-20.

[41] J. Fröhlich, W. Rodi, Introduction to large eddy simulation of turbulent flows, Closure strategies for turbulent and transitional flows 1 (8) (2002) $197-224$.

[42] B. J. Geurts, Regularization modeling for large-eddy simulation of diffusion flames.

[43] H. Carslaw, J. Jaeger, Some two-dimensional problems in conduction of heat with circular symmetry, Proceedings of the London mathematical society 2 (1) (1940) 361-388.

[44] F. Bowman, Introduction to Bessel Functions, Dover Publications Inc., 2010.

[45] S. Wan, Z. Yin, K. Zhang, H. Zhang, X. Li, A topology-preserving optimization algorithm for polycube mapping, Computers and Graphics 35 .

[46] W. Yu, K. Zhang, S. Wan, X. Li, Optimizing polycube domain construction for hexahedral remeshing, Computer-Aided Design 46 (2014) $58-68$.

[47] S. Park, T. A. Davis, W. W. Hager, H. Zhang, Quadratic programming techniques for graph partitioning. (2006).

[48] H. Meyerhenke, B. Monien, T. Sauerwald, A new diffusion-based multilevel algorithm for computing graph partitions, J. Parallel Distrib. Comput. 69 (9) (2009) 750-761. 
[49] W. W. Hager, Y. Krylyuk, Graph partitioning and continuous quadratic programming, SIAM Journal on Discrete Mathematics 12 (4) (1999) $500-523$. 